\title{
Discrepancies in the diagnosis of intraductal proliferative lesions of the breast and its management implications: results of a multinational survey
}

\author{
Mohiedean Ghofrani • Beatriz Tapia • \\ Fattaneh A. Tavassoli
}

Received: 10 March 2006 / Accepted: 31 May 2006 / Published online: 13 October 2006

(C) Springer-Verlag 2006

\begin{abstract}
To measure discrepancies in diagnoses and recommendations impacting management of proliferative lesions of the breast, a questionnaire of five problem scenarios was distributed among over 300 practicing pathologists. Of the 230 respondents, $56.5 \%$ considered a partial cribriform proliferation within a duct adjacent to unequivocal ductal carcinoma in situ (DCIS) as atypical ductal hyperplasia (ADH), 37.7\% of whom recommended reexcision if it were at a resection margin. Of the $43.5 \%$ who diagnosed the partially involved duct as DCIS, $28.0 \%$ would not recommend reexcision if the lesion were at a margin. When only five ducts had a partial cribriform proliferation, $35.7 \%$ considered it as DCIS, while if $\geq 20$ ducts were so involved, this figure rose to $60.4 \%$. When one duct with a complete cribriform pattern measured 0.5 , 1.5 , or $4 \mathrm{~mm}$, a diagnosis of DCIS was made by 22.6, 31.3, and $94.8 \%$, respectively. When multiple ducts with flat epithelial atypia were at a margin, $20.9 \%$ recommended reexcision. Much of these discrepancies arise from the artificial separation of $\mathrm{ADH}$ and low-grade DCIS and emphasize the need for combining these two under the umbrella designation of ductal intraepithelial neoplasia grade 1 (DIN 1) to diminish the impact of different terminologies applied to biologically similar lesions.
\end{abstract}

\footnotetext{
M. Ghofrani $\cdot$ B. Tapia $\cdot$ F. A. Tavassoli $(\bowtie)$

Department of Pathology, Yale University School of Medicine, Lauder Hall (LH) 222, 310 Cedar St.,

New Haven, CT 06510, USA

e-mail: Fattaneh.Tavassoli@Yale.edu

Present address:

B. Tapia

Department of Pathology and Immunology,

Washington University School of Medicine,

St. Louis, MO, USA
}

Keywords Breast - Ductal carcinoma in situ - Hyperplasia . Interobserver variability . Ductal intraepithelial neoplasia

\section{Introduction}

The concept of atypical hyperplasia was introduced decades ago within the continuum of intraductal breast proliferations, a continuum which encompasses benign proliferations of usual ductal hyperplasia to high-grade ductal carcinoma in situ (DCIS) [3-5, 7, 15, 32]. The term atypical ductal hyperplasia (ADH) was used initially for a vaguely defined group lesions that had "some but not all of the requisite features of ductal carcinoma in situ" [21]. Subsequently, the qualitative similarity to low-grade DCIS (LG-DCIS) was stressed, and quantitative measures were introduced to separate ADH from DCIS [21, 31].

Totally arbitrary boundaries were drawn to separate ADH from LG-DCIS, a separation that resulted in drastically different management approaches in the 1980s-i.e., mastectomy for those diagnosed as DCIS and follow-up for those with a diagnosis of ADH. Now, even with widespread use of conservative surgery, patient management differs significantly depending on whether a lesion is diagnosed as $\mathrm{ADH}$ or DCIS. The two most notable approaches for this separation have been based on: (a) the definition by Page et al. [21] who defined the minimum requirement for LGDCIS as complete involvement of at least two spaces by a proliferation that cytologically and architecturally resembles DCIS and (b) the definition of Tavassoli and Norris [31] who required complete involvement of one or more ducts by a cribriform or micropapillary proliferation of uniform cells with low-grade nuclei exceeding $2 \mathrm{~mm}$ in aggregate diameter for a diagnosis of LG-DCIS. According to either of these two definitions, any intraductal prolifer- 
ative lesion that demonstrated the qualitative cytologic and architectural features of LG-DCIS but failed to pass the defined quantitative threshold was to be designated as $\mathrm{ADH}$.

Since then, no objective molecular, ultrastructural, immunohistochemical, or morphometric feature has been identified to reliably distinguish ADH from LG-DCIS $[1,11,15,16,18,25]$. Therefore, the distinction of the two continues to be based solely on application of the arbitrary criteria mentioned above. Frequently cited studies in the literature, which have attempted to calculate the relative risk of various proliferative lesions, may have claimed to adhere to the criteria of Page et al. have actually deviated from the two-space rule and have accepted single-space involvement as DCIS, thereby blurring the distinction between levels of hyperplasia and atypia and diminishing the practical utility of such a distinction [8].

We conducted a survey 15 and 20 years postintroduction of these two sets of criteria to determine how much uniformity or discrepancy exists in the interpretation of these lesions and how it would impact current patient management.

\section{Materials and methods}

To investigate pathologists' approach to selected problems in the diagnosis and management of intraductal proliferative lesions of the breast, a questionnaire was prepared with diagrammatic representations of five potentially problematic scenarios in breast pathology dealing with intraductal proliferative lesions (Figs. 1, 2, 3, 4, 5). It was decided to use diagrammatic representations rather than glass slides to facilitate distribution of the survey among a greater number of pathologists and to ensure that the participants focused on the same diagnostic issue, given the presence of certain lesions in the background. The questionnaire also inquired whether the respondents considered breast pathology their area of expertise, the average number of breast biopsies and lumpectomies or mastectomies they signed out each week, the practice setting in which they worked in (academic, community, or private), and how long they had been in practice.

The questionnaire was distributed among over 300 pathologists who were either known for their expertise and specialization in breast pathology in various countries or who were interested in the field as evidenced by their participation in educational courses dedicated to breast pathology. If a participant left a response in the questionnaire blank, that response was considered null and excluded from calculations. Also, for all questions regarding diagnosis and management of lesions illustrated in the questionnaire, the respondents were given the option of choosing one of the two answers (i.e., ADH vs DCIS, Yes vs No). The responses of the small minority who chose both were similarly excluded from analyses. Also, if a participant responded to the questions regarding average number of biopsies or lumpectomies/mastectomies a week by providing a numeric range, the average value of the range was considered for calculations. The collected responses were anonymized and tabulated in a custom Microsoft Access database (Microsoft Corporation, Redmond, WA, USA), and custom queries were written for data analysis. Statistical significance of the results was evaluated using chi-square and $t$ tests.

\section{Results}

A total of 230 completed questionnaires were received and included in the study. Of the 230 respondents, 93 (40.4\%) considered breast pathology their area of expertise, while $130(56.5 \%)$ did not consider themselves experts but had special interest or responsibility for signing out breast pathology. The respondents signed out an average and standard deviation of $10.1 \pm 9.5$ biopsies and 7.5 \pm 7.8 lumpectomies or mastectomies a week. These figures were $14.4 \pm 11.4$ biopsies and $10.3 \pm 9.0$ lumpectomies or mastectomies a week for the responding pathologists who considered breast pathology their area of expertise and $6.9 \pm 6.0$ biopsies and $5.3 \pm 6.0$ lumpectomies or mastectomies a week for those who did not. $T$ test showed the differences between the means in these two groups to be statistically significant $(p<0.0001$ for both biopsies and lumpectomies/mastectomies).

Academic pathologists constituted $85(37.0 \%)$ of the respondents, while $113(49.1 \%)$ of the respondents worked in community hospitals and $20(8.7 \%)$ in private laboratories. Nine pathologists $(3.9 \%)$ worked in more than one practice setting. Sixty-five pathologists $(28.3 \%)$ had been practicing pathology for less than 5 years, 37 (16.1\%) for 6 to 10 years, and $125(54.3 \%)$ had over 10 years of practice experience.

As shown in Fig. 1, the first question addressed whether respondents considered a partially involved duct adjacent to unequivocal cribriform DCIS as ADH or DCIS and, depending on their response, whether they would recommend reexcision if this partially involved duct was less than $0.1 \mathrm{~mm}$ from the excision margin. Of the 230 respondents, $130(56.5 \%)$ considered this partial cribriform proliferation as ADH and, among these respondents, 49 (37.7\%) recommended reexcision of the $\mathrm{ADH}$ if it were close to the excision margin. On the other hand, of the 100 (43.5\%) who diagnosed this partially involved duct as DCIS, 28 (28.0\%) would not recommend reexcision if the lesion were close to the excision margin. Although chi-square analysis showed that pathologists who made a diagnosis of DCIS were significantly more likely 
Fig. 1 The first scenario assessed how pathologists would diagnose a partially involved duct adjacent to unequivocal cribriform DCIS, and whether they would recommend reexcision if it were less than $0.1 \mathrm{~mm}$ from a resection margin

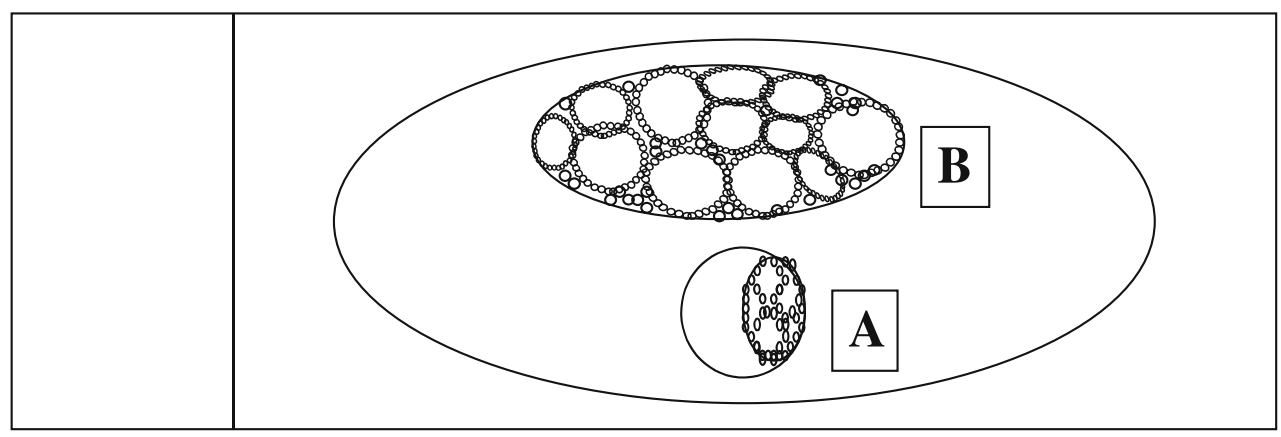

"A" is a single duct with unequivocal cribriform grade 1 DCIS

" $\mathrm{B}$ " is a duct with partial involvement by a cribriform pattern located $<0.1 \mathrm{~mm}$ from the margin.

1. What is your diagnosis for duct " $\mathrm{B}$ "?

DCIS

ADH

2. Would you recommend re-excision?

Yes

No to recommend reexcision compared to those who made a diagnosis of $\mathrm{ADH}(p<0.001)$, the final impact on patient management was that, regardless of the diagnosis, 116 $(50.4 \%)$ would recommend to have a reexcision while 108 $(47.0 \%)$ would not.

Question 2 documented how respondents categorized a partial cribriform proliferation involving a few (five) or numerous (20 or more) ducts (Fig. 2). When only five ducts had a partial cribriform proliferation, $82(35.7 \%)$ of the respondents considered it as DCIS. With $\geq 20$ ducts so involved, $139(60.4 \%)$ of the respondents designated the changes as DCIS. Chi-square testing showed this difference to be statistically significant $(p<0.001)$.
In Question 3, as shown in Fig. 3, the minimum size requirement for diagnosis of LG-DCIS in a single duct was addressed. When a single duct with a complete cribriform pattern measured $0.5,1.5$, or $4 \mathrm{~mm}$, a diagnosis of DCIS was made by $52(22.6 \%), 72(31.3 \%)$, and $218(94.8 \%)$ of the respondents, respectively. Chi-square testing confirmed that pathologists were significantly more likely to diagnose DCIS if the single duct measured $>2 \mathrm{~mm}(p<0.001)$.

Question 4 addressed the approach to management of flat epithelial atypia when it was present in multiple ducts located within less than $0.1 \mathrm{~mm}$ from the excision margin (Fig. 4). In this scenario, when multiple ducts with flat epithelial atypia were close to an excision margin, 48 respondents $(20.9 \%)$ recommended reexcision.

Fig. 2 In question 2, participants were asked whether the number of partially involved ducts affects their decision to make a diagnosis of $\mathrm{ADH}$

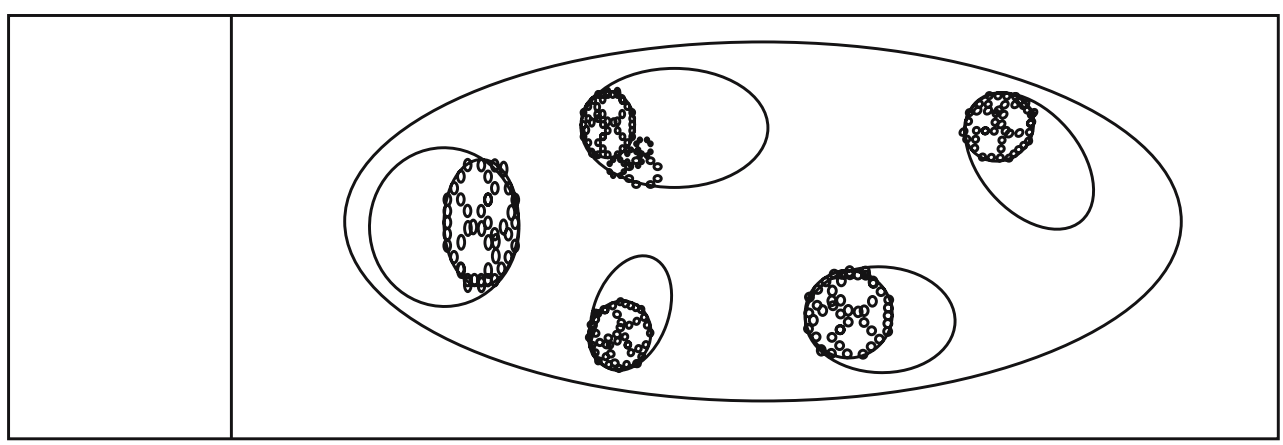

There are multiple ducts (5) with partial cribriform proliferation; there is no necrosis. 1 . What is your diagnosis?

DCIS

$\mathrm{ADH}$

2. What would your diagnosis be if $>20$ ducts showed the same finding?

DCIS 
Fig. 3 Responses to question 3 demonstrated what pathologists thought was the lowest size threshold required to make a diagnosis of DCIS

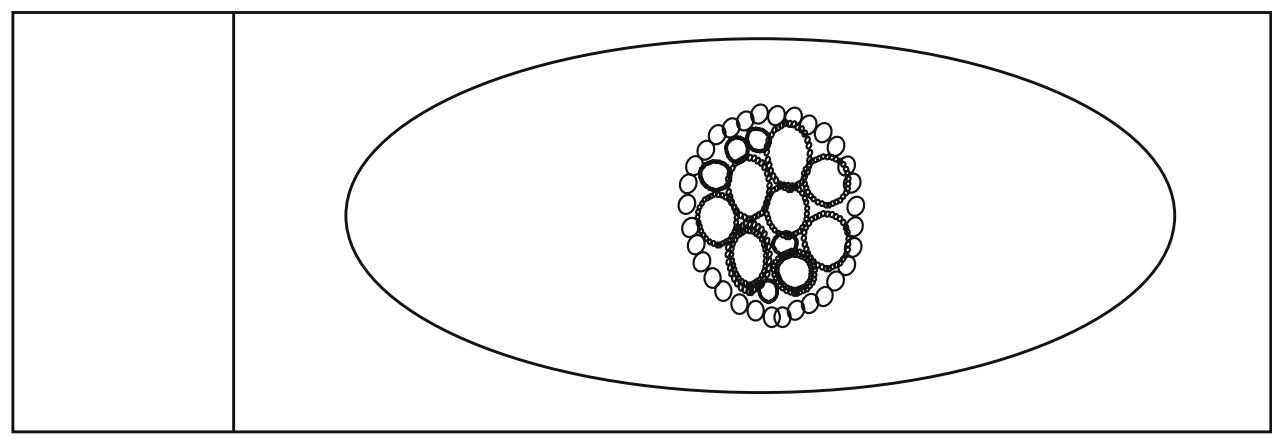

There is a single $1.5 \mathrm{~mm}$ duct with classic cribriform nuclear grade 1 pattern; there is no necrosis.

1. What is your diagnosis?

DCIS

$\mathrm{ADH}$

2. What would your diagnosis be if the duct were $0.5 \mathrm{~mm}$ ?

$$
\text { DCIS }
$$

$\mathrm{ADH}$

3. What would your diagnosis be if the duct were $4 \mathrm{~mm}$ ?

$$
\text { DCIS }
$$

$\mathrm{ADH}$
Finally, question 5 evaluated how respondents measured invasive carcinoma when two foci of stromal microinvasion (measuring less than $1 \mathrm{~mm}$ each) emanated from two opposite poles of a 1-cm duct with high-grade comedo-type DCIS (Fig. 5). Of the 230 respondents, 185 (80.4\%) measured these as two separate foci of microinvasion (less than $1 \mathrm{~mm}$ each), while 37 (16.1\%) considered the total size of invasive carcinoma as the aggregate diameter of the high-grade DCIS plus the two microinvasive foci, namely, $1.19 \mathrm{~cm}$.

Chi-square testing was performed to compare the responses of expert breast pathologists and nonexperts to all five questions. No statistically significant difference was found between the two groups in the proportion of responses to any of the questions.

\section{Discussions}

These results indicate that 15 and 20 years postintroduction of criteria for separation of ADH from DCIS [21, 31], interobserver variability in the diagnosis of intraductal proliferative lesions of the breast has not diminished. Given the significance of margin involvement or proximity in current management of DCIS, this survey demonstrates an
Fig. 4 Question 4 evaluated how participants would manage flat epithelial atypia close to a resection margin

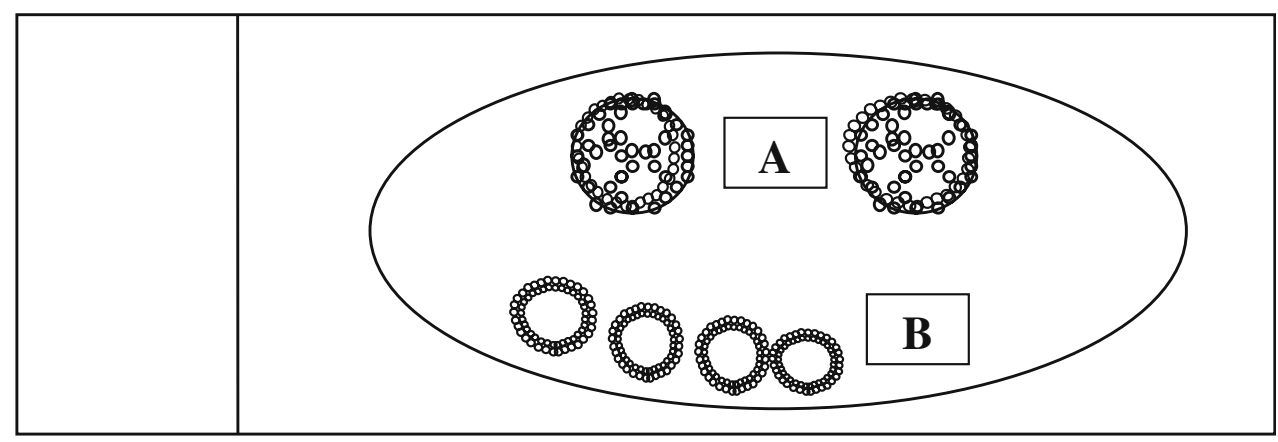

The cribriform grade 1 DCIS (A) is more than $5 \mathrm{~mm}$ from the inked margin.

Multiple ducts with flat epithelial atypia are within $<0.1 \mathrm{~mm}$ of the inked margin (B).

Would you recommend re-excision?

$$
\text { Yes }
$$


Fig. 5 Question 5 surveyed how pathologists measured invasive carcinoma in the presence of multifocal microinvasion

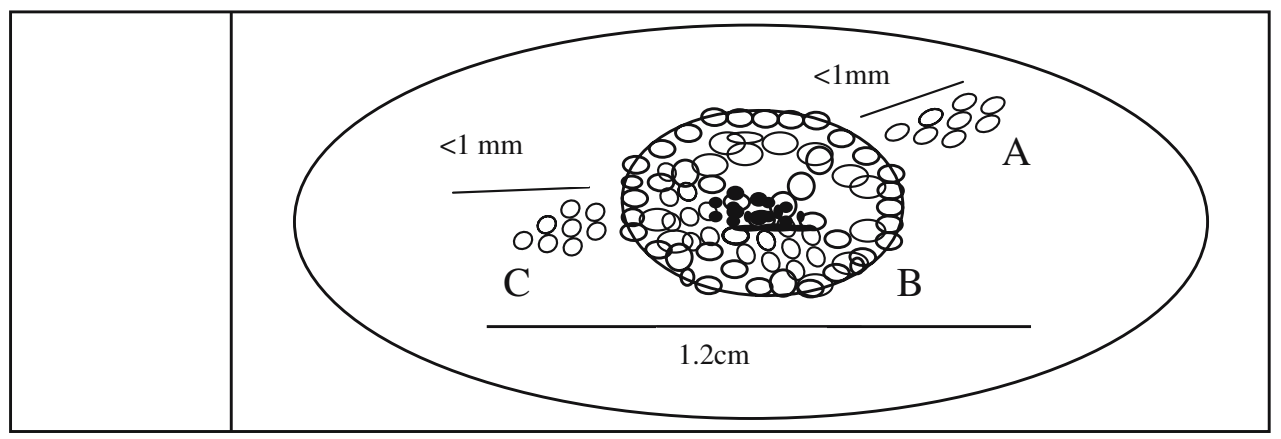

There is a single duct (B) with high grade DCIS and comedo-necrosis and two unequivocal foci of stomal invasion, each $<1 \mathrm{~mm}$, at opposite poles of the duct (A and C) 1. The size of the invasive carcinoma is:

Two separate foci of microinvasion, $<1 \mathrm{~mm}$ each (A and C) The sum of $\mathrm{A}+\mathrm{B}+\mathrm{C}$ (approximately $1.2 \mathrm{~cm}$ ) even wider variability that exists in the management of such lesions and ultimately patient care.

In a survey done by Rosai in 1991, 17 ductal and lobular proliferative breast lesions were distributed among five experts in breast pathology [24]. The participants were asked to categorize such lesions, which had already been circled on glass slides, as either hyperplasia, atypical hyperplasia, carcinoma in situ, or "other" (to be specified) based on the diagnostic criteria they used in their daily practice. In that study, there was no a single case in which all five pathologists agreed on the diagnosis, and there were only three cases $(18 \%)$ in which four of the five pathologists agreed. Also, some pathologists tended to make more malignant diagnoses than others. In his report, Rosai considered this interobserver variability to be unacceptably high and suggested the adoption of a terminology such as mammary intraepithelial neoplasia with a grading system similar to that which was being used for the uterine cervix.

A possible explanation for such a high degree of interobserver variability set forth in that report was that the pathologists were not using a standard set of criteria. Therefore, a year later, Schnitt et al. [27] tried a different approach to assess interobserver variability in the diagnosis of intraductal proliferative lesions of the breast. In their 1992 survey, they asked six experts in breast pathology to evaluate 24 proliferative ductal lesions. In this survey, the participating pathologists agreed to use the criteria of Page et al., and 15 teaching slides representing classic examples of usual ductal hyperplasia, atypical ductal hyperplasia, and noncomedo DCIS were circulated among the six pathologists to foster concordance before initiation of the study. The specific area of interest on each study slide was indicated by masking all the surrounding tissue so that all the participants focused on the same lesion and to prevent any bias that may result from assessment of changes in the surrounding breast tissue. The participants in this survey were asked to adhere to the provided criteria rather than the ones they used in their daily practice. Despite all these efforts, there was complete agreement among all six pathologists in only $14(58 \%)$ of the 24 cases and among five or more pathologists in 17 cases (71\%). The most common diagnostic problem was the distinction of atypical hyperplasia from DCIS in six cases. Although this study showed a significant improvement in interobserver agreement compared to Rosai's survey, the persistence of significant differences among expert breast pathologists even under optimal and highly artificial conditions conveyed a more widespread problem in the pathology community with potential impact on patient management.

Responses to the current survey also demonstrate that although criteria to distinguish ADH from DCIS have been introduced over two decades ago, there is still no agreement on how to categorize these two types of mammary intraductal proliferation. While the sole partially involved duct described in question 1 would not qualify as DCIS according to the criteria set forth by either Page et al. or Tavassoli and Norris, 43.5\% of practicing pathologists, nevertheless, diagnosed it as DCIS. This might seem logical because the partially involved duct probably reflects an extension of the same process present in the nearby unequivocal cribriform DCIS. However, the fact remains that there is no consensus on what to designate a partially involved duct adjacent to unequivocal LG-DCIS and how to manage such a lesion when it is near or on the surgical excision margin [10]. Even among the respondents who considered the partially involved duct in question 1 as $\mathrm{ADH}$, more than a third recommended reexcision if it were close to the margin of resection, and of those who considered this partially involved duct as DCIS, close to $30 \%$ would not recommend reexcision even if the in situ carcinoma were within $0.1 \mathrm{~mm}$ of the resection margin. 
This interobserver variability in diagnosis and management was so high that, in the end, patients would have an almost 50:50 chance of having a reexcision or not, regardless of the diagnosis of the partially involved duct as ADH or DCIS. This was due to a combination of the high proportion diagnosing it as DCIS and the high proportion of those who recommended reexcision even though they interpreted it as ADH. Obviously, the impact of this issue goes beyond simply margin involvement; it is also crucial in the assessment of lesion size, resulting in widely varied assessment of size/extent of LG-DCIS.

Question 2 addressed the concept of extensive ADH. Cognizant of the fact that available criteria require complete involvement of duct cross sections for a diagnosis of lowgrade (cribriform or micropapillary) variants of DCIS, it was surprising to find that even when only five ducts were involved by a partial cribriform proliferation, over $35 \%$ of the respondents considered it as DCIS. Furthermore, when greater than 20 ducts were partially involved by such a cribriform proliferation, the proportion of respondents who treated it as DCIS rose significantly to over $60 \%$. Chisquare testing showed that as the number of partially involved ducts increased, the number of patients diagnosed with cancer significantly increased. Nevertheless, even with drastic differences in the number of involved ducts (5 vs $\geq 20$ ), there was no unanimity in the diagnosis of carcinoma in situ vs atypical hyperplasia.

This scenario has become increasingly more frequent since the introduction of screening mammography. It was not addressed in the criteria introduced by either Page et al. or Tavassoli and Norris because both those retrospective studies were based on predominantly premammographic era biopsies. With the increasing number of such "extensive ADH" cases ( $\geq 20$ partially involved ducts) seen in her consultation practice, Tavassoli suggested that even when these are designated as extensive $\mathrm{ADH}$ due to strict adherence to previously defined criteria, they should be managed as DCIS and reexcised if close to or at the margin of resection [29].

Question 3 demonstrated that the majority of respondents used the 2-mm size criterion rather than the two spaces in diagnosing DCIS. When the single duct described in the question measured greater than $2 \mathrm{~mm}(4 \mathrm{~mm})$, close to $95 \%$ of the respondents considered it DCIS. However, if this single duct measured less than $2 \mathrm{~mm}$, i.e., 0.5 or $1.5 \mathrm{~mm}$, 22.6 and $31.3 \%$ of the respondents considered it DCIS, respectively. Although this difference in the probability of diagnosing DCIS when the lesion measured less than or greater than $2 \mathrm{~mm}$ was statistically significant, it is interesting to note that over $30 \%$ of the respondents considered a single duct measuring $1.5 \mathrm{~mm}$ as DCIS and over $20 \%$ designated a single duct measuring only $0.5 \mathrm{~mm}$ as carcinoma in situ despite the fact that these two scenarios meet neither the criteria of Page et al., which require two completely involved spaces not otherwise specified as to size [21], nor the criteria of Tavassoli and Norris, which require one or more spaces greater than $2 \mathrm{~mm}$ in maximal cross-sectional diameter [31]. Even with a substantial $4 \mathrm{~mm}$ duct, $5.2 \%$ did not designate the lesion as DCIS presumably because of the two-space requirement of Page et al. These responses demonstrate that in the current day to day practice of pathology, some women with a $0.5-\mathrm{mm}$ lesion would be diagnosed as having DCIS, with a reexcision if it were close to or at the resection margin, and most probably radiation therapy, whereas some women with $4 \mathrm{~mm}$ lesions interpreted as ADH would get no reexcision, would be simply followed up, or at most would enter some form of prevention trial with hormone therapy $[2,9]$.

Question 4 in our survey documented the confusion that exists regarding the management of flat epithelial atypia, with over $20 \%$ of respondents recommending reexcision if the lesion were close to an excision margin. As a result, over a fifth of the patients would have reexcision, while the remaining $80 \%$ would not.

The last question showed the various approaches pathologists take in measuring invasive carcinoma when multifocal early invasion emanating from a single duct is present. Although over $80 \%$ of respondents would consider foci of invasion less than $1 \mathrm{~mm}$ emanating from opposite poles of a duct with DCIS as microinvasion, slightly over $16 \%$ of respondents would measure DCIS with its associated microinvasion from opposite poles as one continuous invasive carcinoma, which would entail a drastically different treatment approach.

The results of this survey raise numerous questions about studies performed in different countries and even different institutions within the same country regarding risk factors, treatment, prognosis, and outcome of intraductal proliferative lesions of the breast, which include LG-DCIS and/or ADH-a significant proportion of mammographically detected noninvasive lesions. Even when the criteria used are explicitly stated, application of criteria varies remarkably among pathologists and from one study to the next. Certainly, the issues raised in this study are not uncommon but have not been specifically addressed in any of the major single-, multiinstitutional, or multinational studies on DCIS cases that include LG-DCIS. Most if not all such studies lack a central review of the diagnosis and even many rely on multiple pathologists at sometimes multiple institutions. This study also illustrates that 15 to 20 years of education of pathologists at local, national, and international courses has not helped much in increasing the level of agreement and uniformity in the diagnosis and interpretation of these common lesions using the criteria available for separating ADH from LG-DCIS. Chi-square 
testing failed to reveal any statistically significant difference in the response behavior of expert breast pathologists and pathologists who did not consider themselves experts.

Since the institution of widespread screening mammography, the number of early, LG-DCIS lesions that are diagnosed has dramatically increased [13, 14, 19]. How partially involved ducts, often spread around completely involved ducts, are interpreted could change the size/extent of the lesion by several centimeters. This potentially undermines the internal consistency and comparability of epidemiological studies and clinical trials regarding such lesions.

In his 1991 survey, Rosai lamented the fact that there were no known morphometric, ultrastructural, immunohistochemical, or molecular features to distinguish $\mathrm{ADH}$ from LG-DCIS [24]. Unfortunately, this issue continues to be the case $[1,11,15,16,18,25]$. Pathologists must assign lesions within the ADH-DCIS continuum to one end of the spectrum or the other based on the morphologic features present on an H\&E-stained slide and an arbitrary set of quantitative criteria that cannot be applied to every lesion encountered. Not only the subjective nature of interpreting the morphologic findings but also the existence of different and in some cases conflicting diagnostic criteria create considerable interobserver variability in distinguishing ADH from LG-DCIS.

This diagnostic variability in turn leads to confusion regarding the optimal management approach to such intraductal proliferative lesions because a diagnosis of LG-DCIS is automatically associated with a significantly worse prognosis and usually requires a more drastic surgical approach compared to ADH. Furthermore, the negative impact of the diagnosis of "carcinoma", albeit an in situ one, on patients' psychological well-being (depression and anxiety) has been well documented [12, 23] and should be a consideration in the choice of optimal terminology.

Molecular, immunohistochemical, and morphologic similarities indicate that the only difference between $\mathrm{ADH}$ and LG-DCIS is a quantitative one $[1,11,15,16$, $18,25]$. The claim that separation of ADH from LG-DCIS is justified based on the development of carcinomas post$\mathrm{ADH}$ in either breast while those that develop after LGDCIS occur in the same breast is questionable [20, 22, 26]. Over a period of 23 years, three papers have appeared on this topic based on a group of 28 women with a median follow-up of 31 years, noting that the invasive carcinomas that occur after LG-DCIS treated by biopsy alone develop in the same breast and in the same quadrant from which the original biopsy with DCIS was taken. Seven of the 11 women who developed subsequent invasive carcinoma were diagnosed within 10 years of the DCIS biopsy, 1 was diagnosed within 12 years, and the remaining 3 developed infiltrating carcinomas over 23-42 years. The authors conclude that these results "indicate a striking dividing point biologically and histopathologically between LG-DCIS lesions and the cytologically similar but lesser lesions of ADH" [26]. When 7 of the 28 women developed invasive carcinoma within 10 years, one wonders why the remaining patients were not contacted to receive appropriate therapy for their disease rather than continuing the study for another 20 years.

Judging from the microscopic description and the sampling documented for these 28 cases, a substantial number of these lesions were probably not low grade because they had some atypia and/or necrosis, albeit not diffusely, suggesting that those who died within 10 years most likely had substantial amount of residual disease and/or higher grade DCIS lesions [6]. The gross and pathologic features of these 28 cases are described in detail in the initial paper published in 1982, which did not refer to them as low grade [20]. Therefore, it is more likely that this study reflects the natural history of a wide variety of intraductal carcinomas, only some of which were LGDCIS. A more simple explanation for any differences that might exist between ADH and LG-DCIS in the frequency of subsequent progression would be that a minuscule lesion, once totally removed, results in near equalization of the risk for subsequent development of carcinoma in the two breasts of that individual patient. A more extensive process, however, is less likely to be completely eliminated by conservative surgical excision alone, leading to the development of recurrences at the same site due to residual disease [6].

A recent epidemiological study has shown a nearly equal incidence rate (5.4 vs 4.5/1,000 person-years) for development of subsequent invasive carcinoma in either breast after a diagnosis of DCIS among close to 37,700 patients diagnosed between January 1988 and December 2002, many of whom were treated by surgery and radiation therapy [17]. This confirms the fact that once a DCIS is adequately treated, it results in equalization of risk for either breast. Furthermore, it indicates that complete excision is an adequate therapy for small lesions. Therefore, complete excision with adequate margins should be explored further as the only therapy for small DIN 1 (DCIS grade 1) lesions that do not exceed 3-4 $\mathrm{mm}$ in maximum extent, particularly when there is no evidence of even any flat epithelial atypia around the DCIS.

It seems only logical to adopt a terminology in which two morphologically identical lesions are not given diagnostic designations as different as "hyperplasia" and "carcinoma". The use of the DIN terminology may help solve this problem $[28,30]$. Although the DIN terminology does not claim to reduce interobserver variability, it 
diminishes the effect of drastically different designations for similar lesions by including the spectrum of atypical ductal hyperplasia and LG-DCIS under the umbrella designation of grade 1 DIN.

\section{References}

1. Allred DC, O'Connell P, Fuqua SA, Osborne CK (1994) Immunohistochemical studies of early breast cancer evolution. Breast Cancer Res Treat 32:13-18

2. Arpino G, Laucirica R, Elledge RM (2005) Premalignant and in situ breast disease: biology and clinical implications. Ann Intern Med 143(6):446-457

3. Ashikari R, Huvos AG, Snyder RE, Lucas JC, Hutter RV, McDivitt RW, Schottenfeld D (1974) A clinicopathologic study of atypical lesions of the breast. Cancer 33:310-317

4. Ashikari R, Huvos AG, Snyder RE, Sharma R, Kirch R, Schottenfeld D (1980) A clinicopathologic study of atypical lesions of the breast further follow up. Pathol Res Pract $166: 481-490$

5. Azzopardi JG (1979) Problems in breast pathology. Saunders, Philadelphia

6. Bellamy CO, McDonald C, Salter DM, Chetty U, Anderson TJ (1993) Noninvasive ductal carcinoma of the breast: the relevance of histologic categorization. Hum Pathol 24:16-23

7. Black MM, Barclay TH, Cutler SJ, Hankey BF, Asire AJ (1972) Association of atypical characteristics of benign breast lesions with subsequent risk of breast cancer. Cancer 29:338-343

8. Bodian CA, Perzin KH, Lattes R, Hoffmann P, Abernathy TG (1993) Prognostic significance of benign proliferative breast disease. Cancer 71:3896-3907

9. Burstein HJ, Polyak K, Wong JS, Lester SC, Kaelin CM (2004) Ductal carcinoma in situ of the breast. $\mathrm{N}$ Engl J Med 350:1430-1441

10. Ceilley E, Jagsi R, Goldberg S, Kachnic L, Powell S, Taghian A (2004) The management of ductal carcinoma in situ in North America and Europe: results of a survey. Cancer 101:1958-1967

11. Chuaqui RF, Zhuang Z, Emmert-Buck MR, Liotta LA, Merino MJ (1997) Analysis of loss of heterozygosity on chromosome 11q13 in atypical ductal hyperplasia and in situ carcinoma of the breast. Am J Pathol 150:297-303

12. De Morgan S, Redman S, White KJ, Cakir B, Boyages J (2002) "Well, have I got cancer or haven't I?" The psycho-social issues for women diagnosed with ductal carcinoma in situ. Health Expect 5:310-318

13. Ernster VL, Barclay J (1997) Increases in ductal carcinoma in situ (DCIS) of the breast in relation to mammography: a dilemma. J Natl Cancer Inst Monogr 22:151-156

14. Ernster VL, Ballard-Barbash R, Barlow WE, Zheng Y, Weaver DL, Cutter G, Yankaskas BC, Rosenberg R, Carney PA, Kerlikowske K, Taplin SH, Urban N, Geller BM (2002) Detection of ductal carcinoma in situ in women undergoing screening mammography. J Natl Cancer Inst 94:1546-1554

15. Goldenberg VE, Goldenberg NS, Sommers SC (1969) Comparative ultrastructure of atypical ductal hyperplasia, intraductal carcinoma, and infiltrating ductal carcinoma of the breast. Cancer 24:1152-1169
16. Lakhani SR, Collins N, Stratton MR, Sloane JP (1995) Atypical ductal hyperplasia of the breast: clonal proliferation with loss of heterozygosity on chromosomes $16 \mathrm{q}$ and $17 \mathrm{p}$. J Clin Pathol 48:611-615

17. Li CI, Malone KE, Saltzman BS, Daling JR (2006) Risk of invasive breast carcinoma among women diagnosed with ductal carcinoma in situ and lobular carcinoma in situ, 1988-2001. Cancer 106:2104-2112

18. O'Connell P, Pekkel V, Fuqua SA, Osborne CK, Clark GM, Allred DC (1998) Analysis of loss of heterozygosity in 399 premalignant breast lesions at 15 genetic loci. J Natl Cancer Inst 90:697-703

19. Osteen RT, Cady B, Chmiel JS, Clive RE, Doggett RL, Friedman MA, Hussey DH, Kraybill WG, Urist MM, Winchester DP (1994) 1991 national survey of carcinoma of the breast by the Commission on Cancer. J Am Coll Surg 178:213-219

20. Page DL, Dupont WD, Rogers LW, Landenberger M (1982) Intraductal carcinoma of the breast: follow-up after biopsy only. Cancer 49:751-758

21. Page DL, Dupont WD, Rogers LW, Rados MS (1985) Atypical hyperplastic lesions of the female breast: a long-term follow-up study. Cancer 55:2698-2708

22. Page DL, Dupont WD, Rogers LW, Jensen RA, Schuyler PA (1995) Continued local recurrence of carcinoma 15-25 years after a diagnosis of low grade ductal carcinoma in situ of the breast treated only by biopsy. Cancer 76:1197-1200

23. Rakovitch E, Franssen E, Kim J, Ackerman I, Pignol JP, Paszat L, Pritchard KI, Ho C, Redelmeier DA (2003) A comparison of risk perception and psychological morbidity in women with ductal carcinoma in situ and early invasive breast cancer. Breast Cancer Res Treat 77:285-293

24. Rosai J (1991) Borderline epithelial lesions of the breast. Am J Surg Pathol 15:209-221

25. Ruiz A, Almenar S, Callaghan RC, Llombart-Bosch A (1999) Benign, preinvasive and invasive ductal breast lesions. A comparative study with quantitative techniques: morphometry, image- and flow cytometry. Pathol Res Pract 195:741-746

26. Sanders ME, Schuyler PA, Dupont WD, Page DL (2005) The natural history of low-grade ductal carcinoma in situ of the breast in women treated by biopsy only revealed over 30 years of longterm follow-up. Cancer 103:2481-2484

27. Schnitt SJ, Connolly JL, Tavassoli FA, Fechner RE, Kempson RL, Gelman R, Page DL (1992) Interobserver reproducibility in the diagnosis of ductal proliferative breast lesions using standardized criteria. Am J Surg Pathol 16:1133-1143

28. Tavassoli FA (1998) Ductal carcinoma in situ: introduction of the concept of ductal intraepithelial neoplasia. Mod Pathol 11:140-154

29. Tavassoli FA (1999) Pathology of the breast, 2nd edn. Appleton \& Lange, Stamford, CT

30. Tavassoli FA (2005) Breast pathology: rationale for adopting the ductal intraepithelial neoplasia (DIN) classification. Nat Clin Pract Oncol 2:116-117

31. Tavassoli FA, Norris HJ (1990) A comparison of the results of long-term follow-up for atypical intraductal hyperplasia and intraductal hyperplasia of the breast. Cancer 65:518-529

32. Wellings SR, Jensen HM, Marcum RG (1975) An atlas of subgross pathology of the human breast with special reference to possible precancerous lesions. J Natl Cancer Inst 55:231273 